\title{
Analysis of the susceptibility in a fluid system with Neumann - plus boundary conditions
}

\author{
Peter Djondjorov ${ }^{1, \star}$, Vassil Vassilev ${ }^{1, \star \star}$, and Daniel Dantchev ${ }^{1, \star \star \star}$ \\ ${ }^{1}$ Institute of Mechanics, Bulgarian Academy of Sciences, Acad. Georgi Bonchev Street, Block 4, 1113 Sofia, \\ Bulgaria.
}

\begin{abstract}
The behaviour of the local and total susceptibilities of a fluid system bounded by different surfaces is studied in the framework of the Ginsburg-Landau Ising type model. The case of a plain geometry, Neumann-infinity boundary conditions under variations of the temperature and an external ordering field is considered. Exact analytic expressions for the order parameter, local and total susceptibilities in such a system are presented. They are used to analyse the phase behaviour of fluids confined in regions close to the bulk critical point of the respective infinite system.
\end{abstract}

\section{Introduction}

It is well known that the phase behaviour of a fluid system (simple, or mixture) limited in its spatial extends depends on its interaction with material bodies bounding it, immersed in and/or surrounding this fluid system. In general, this phase behaviour essentially differs from that of the infinite, unbounded, system. On the other hand, from a practical point of view, the phase behaviour of a fluid system is important for the stability and consistency of various devices immersed in it, especially for micro- and nano-technologies.

This contribution concerns the behaviour of fluid systems which has a limited spatial extend in one dimension and forming a film geometry $\infty^{2} \times L$ ( $L$ being the characteristic length of the system), including the fluid mediated interactions between the boundaries of the system.

In the vicinity of the bulk critical temperature $T_{c}$ of the bulk system, one observes a diversity of surface phase transitions [1,2] of different kind in which the surface orders before, together, or after ordering in the bulk of the system, which are known as normal (or extraordinary), surface-bulk and ordinary surface phase transitions. For a simple fluid or for binary liquid mixtures the wall generically prefers one of the fluid phases or one of the components. In the vicinity of the bulk critical point the last leads to the phenomenon of critical adsorption [3-13]. This can be modelled by considering local surface field $h_{1}$ acting solely on the surfaces of the system. When the system undergoes a phase transition in its bulk in the presence of such surface ordering fields one speaks about the "normal" transition [14]. It has been shown that it is equivalent, as far as the leading critical behaviour is concerned, to the "extraordinary" transition $[12,14]$ which is achieved by enhancing the

\footnotetext{
^e-mail: padjon@imbm.bas.bg

$\star \star$ e-mail: vasivas@imbm.bas.bg

$\star \star \star$ e-mail: danieldantchev@gmail.com
} 
surface couplings to become stronger enough than the bulk couplings. In the remainder of this article we will use the surface field picture. There is also a special case of surface enrichment, when the surface orders simultaneously with the bulk. This is normally termed surface-bulk, or special phase transition. One way to achieve it is to enhance the coupling on the surface and very near to it, so that one achieves the delicate balance needed to get the both orderings to become simultaneously. On a mean field level this can be described by imposing Neumann boundary conditions on the boundary.

In the current article a generic mean-field model system in which on one surface the system is subjected to strong surface adsorption, while the other is such that the surface orders exactly at the same temperature as the bulk, is considered. Mathematically, within the continuum mean-field approach such a system is considered to be characterized with the (Neumann, + ) boundary conditions, where the + signs means that the corresponding order parameter tends to $+\infty$ at the boundary with strong adsorption. This type of boundary conditions can be achieved on a magnetic system. In principle, such boundary conditions can be implemented for a fluid system as well, but an extremely precise arrangement for one of the surfaces has to be performed.

In the current work the order parameter profile and its response functions to an externally applied ordering field $h$, as well as the free energy will be studied as functions of both the temperature $T$ and the field $h$ within the three-dimensional continuum mean-field Ising model. Analytical results for the $h$-dependence of the model in the form of the full $(T ; h)$ dependences of the order parameter, local and total susceptibilities in the $(T ; h)$ plane are presented for the case of above mentioned Neumann plus infinity boundary conditions.

\section{Ginzburg-Landau mean-field model}

\subsection{Basic definition of the problem considered}

A critical system of Ising type in a film geometry $\infty^{2} \times L$ is considered, where $L$ is supposed to be along the $z$ axis. Its state is described by the minimizers of the standard $\phi^{4}$ Ginzburg-Landau functional

$$
\mathcal{F}[\phi ; \tau, h, L]=\int_{0}^{L} \mathcal{L}\left(\phi, \phi^{\prime}\right) \mathrm{d} z,
$$

with

$$
\mathcal{L}\left(\phi, \phi^{\prime}\right)=\frac{1}{2} \phi^{\prime 2}+\frac{1}{2} \tau \phi^{2}+\frac{1}{4} g \phi^{4}-h \phi,
$$

where $\phi(z ; \tau, h, L)$ is the order parameter, $\tau=\left(T-T_{c}\right) /\left[T_{c}\left(\xi_{0}^{+}\right)^{-2}\right]$ is the bare reduced temperature with $\xi_{0}^{+}$being the nonuniversal amplitude of the correlation length along the temperature axis, $h$ is the external ordering field, $g$ is the bare coupling constant and the primes indicate differentiation with respect to the variable $z$.

\subsection{Euler-Lagrange equation for the order parameter}

The extremals of the functional $\mathcal{F}$ are determined by the solutions of the corresponding EulerLagrange equation

$$
\frac{d}{d z} \frac{\partial \mathcal{L}}{\partial \phi^{\prime}}-\frac{\partial \mathcal{L}}{\partial \phi}=0,
$$

which, on account of Eq. (2), reads

$$
\phi^{\prime \prime}-\phi\left[\tau+g \phi^{2}\right]+h=0 .
$$


Multiplying the above equation by $\phi^{\prime}$ and integrating once over $z$, one obtains the following first integral of the system

$$
\frac{1}{2} \phi^{\prime 2}=\frac{1}{2} \tau \phi^{2}+\frac{1}{4} g \phi^{4}-h \phi+c=P[\phi]
$$

where $c$ is the constant of integration. The value of the integration constant $c$ determines three types of roots of the polynomial $P[\phi]$ - four real roots, two real and two complex conjugated roots (because the coefficients of $P[\phi]$ are real numbers) and two couples of complex conjugated roots as well as various combinations of multiple roots.

\subsection{Local and total susceptibilities}

By definition, the local layered susceptibility in the case under consideration is

$$
\chi_{l}(z \mid \tau, h) \equiv \frac{\mathrm{d} \phi(z \mid \tau, h)}{\mathrm{d} h}=\frac{\partial \phi(z \mid \tau, h)}{\partial h}+\frac{\partial \phi(z \mid \tau, h)}{\partial x_{0}} \frac{\partial x_{0}}{\partial h},
$$

where $\mathrm{d} a / \mathrm{d} h$ stands for the total derivative of the function $a$ with respect to the variable $h$. Differentiating all terms in Eq. (4) with respect to $h$, one obtains an equation for $\chi_{l}$ of form

$$
-\chi_{l}^{\prime \prime}+\left(\tau+3 g \phi^{2}\right) \chi_{l}=1
$$

Based on the knowledge of the local susceptibility, one can determine the total susceptibility $\chi_{T}\left(x_{t}, x_{h}\right)$ simply by integrating

$$
\chi_{T}\left(x_{t}, x_{h}\right)=\frac{1}{L} \int_{0}^{L} \chi_{l}(z \mid t, h) d z
$$

\section{Analytical expressions}

\subsection{Explicit expressions for the order parameter}

Hereafter we specialise to boundary conditions of form

$$
\phi^{\prime}(0)=0, \quad \phi(L)=+\infty
$$

that is of Neumann type on the left boundary and $+\infty$ on the right one. We are looking for real-valued solutions of Eq. (5), corresponding to given values of the parameters $\tau$ and $h$, which are smooth in the open interval $(0,1)$, and satisfy the Neumann-infinity boundary conditions $(9)$. In the case of $(+\infty,+\infty)$ boundary conditions, analytical results for the order parameter and the local and total susceptibilities are presented in our previous paper [15]. Using the results in that paper, we are going to obtain solutions for the case of Neumann-infinity boundary conditions by an appropriate scaling of the results in [15]. Thus, in terms of the scaling variables

$$
\zeta=z / L, \quad x_{t}=\tau L^{1 / v}, \quad x_{h}=\sqrt{2 g} h L^{\Delta / v}, \quad \phi(z)=\sqrt{\frac{2}{g}} L^{-\beta / v} X_{m}\left(\zeta \mid x_{t}, x_{h}\right),
$$

with $\beta=v=1 / 2$ and $\Delta=3 / 2$, Eq. (4) for the order parameter takes the form

$$
X_{m}^{\prime \prime}(\zeta)=X_{m}(\zeta)\left[x_{t}+2 X_{m}^{2}(\zeta)\right]-\frac{x_{h}}{2}
$$


In the cases when there exist multiple real-valued solutions that meet the differential equation (11) and boundary conditions (9), we seek the one that provides minimal value of the energy functional

$$
\mathcal{E}=\int_{0}^{1} f\left(X_{m}(\zeta), X_{m}^{\prime}(\zeta)\right) d \zeta
$$

where

$$
f\left(X_{m}(\zeta), X_{m}^{\prime}(\zeta)\right)=\left[X_{m}^{\prime}(\zeta)\right]^{2}+X_{m}^{4}(\zeta)+x_{t} X_{m}^{2}(\zeta)-x_{h} X_{m}(\zeta)
$$

is the energy density. In Eq. (11) and below, the primes indicate differentiation with respect to the variable $\zeta$ which, as follows from Eq. (10), varies in the closed interval [0,1]. According to Eq. (5) the first integral of Eq. (11) reads

$$
\left[X_{m}^{\prime}(\zeta)\right]^{2}=P\left[X_{m}\right], \quad P\left[X_{m}\right]=X_{m}^{4}(\zeta)+x_{t} X_{m}^{2}(\zeta)-x_{h} X_{m}(\zeta)+\varepsilon,
$$

where $\varepsilon$ denotes the respective constant of integration.

First, let us recall that we are interested in real-valued solutions $X_{m}(\zeta)$ of Eq. (11), corresponding to given values of the parameters $x_{t}$ and $x_{h}$, which are smooth in the open interval $(0,1)$ and satisfy the Neumann-infinity boundary conditions. The first boundary condition (9) implies that $P\left(X_{m}(0)\right)=0$. Denoting $X_{m}(0)=X_{m 0}$, we can express the integration constant in the form

$$
\varepsilon=-X_{m 0}\left(X_{m 0}^{3}+x_{t} X_{m 0}-x_{h}\right)
$$

Now, given values of the parameters $x_{t}, x_{h}$ and $X_{m 0}$, each real-valued solution $X_{m}(\zeta)$ of Eq. (14) can be expressed, following $[16, \S 20.22, \S 21.73]$, in the form

$$
X_{m}\left(\zeta \mid x_{t}, x_{h}, X_{m 0}\right)=X_{m 0}+\frac{6 X_{m 0}\left(x_{t}+2 X_{m 0}^{2}\right)-3 x_{h}}{12 \wp\left(\zeta ; g_{2}, g_{3}\right)-\left(x_{t}+6 X_{m 0}^{2}\right)} .
$$

Here, $\wp\left(\zeta ; g_{2}, g_{3}\right)$ is the Weierstrass elliptic function, $g_{2}$ and $g_{3}$ are the invariants of the polynomial $P\left[X_{m}\right]$, which according to $[16, \S 20.22, \S 21.73]$ and Eq. (15) read

$$
\begin{gathered}
g_{2}=\frac{x_{t}}{12}+X_{m 0}\left(x_{h}-x_{t} X_{m 0}-X_{m 0}^{3}\right), \\
g_{3}=-\frac{1}{432}\left(2 x_{t}^{3}+9\left(3 x_{h}^{2}-8 x_{t} x_{h} X_{m 0}+8 x_{t} X_{m 0}^{2}\left(x_{t}+X_{m 0}^{2}\right)\right)\right) .
\end{gathered}
$$

In the case of parameters $\left(x_{t}, x_{h}\right)$ such that more than one solution of form (16) exist, than the order profile of minimal energy (12) is the physical one.

\subsection{Explicit expression for the local and total susceptibilities}

In scaling variables (10) the local susceptibility (6) reads

$$
\chi_{l}\left(z \mid x_{t}, x_{h}\right)=L^{\gamma / v} X_{\chi}\left(\zeta \mid x_{t}, x_{h}\right)
$$

where

$$
X_{\chi}\left(\zeta \mid x_{t}, x_{h}\right)=2 \frac{\mathrm{d}}{\mathrm{d} x_{h}} X_{m}\left(\zeta \mid x_{t}, x_{h}\right)
$$


Then, denoting by $\dot{X}_{m 0}\left(x_{h}\right)$ the partial derivative of $X_{m 0}$ with respect to $x_{h}$, Eq. (6) can be written in the form

$$
X_{\chi}\left(\zeta \mid x_{t}, x_{h}, X_{m 0}\right)=2 \frac{\partial}{\partial x_{h}} X_{m}\left(\zeta \mid x_{t}, x_{h}, X_{m 0}\right)+2 \dot{X}_{m 0}\left(x_{h}\right) \frac{\partial}{\partial X_{m 0}} X_{m}\left(\zeta \mid x_{t}, x_{h}, X_{m 0}\right),
$$

and since $X_{\chi}\left(1 \mid x_{t}, x_{h}, X_{m 0}\right)=0$, this equation implies

$$
\dot{X}_{m 0}\left(x_{h}\right)=-\frac{\frac{\partial}{\partial x_{h}} X_{m}\left(1 \mid x_{t}, x_{h}, X_{m 0}\right)}{\frac{\partial}{\partial X_{m 0}} X_{m}\left(1 \mid x_{t}, x_{h}, X_{m 0}\right)} .
$$

Thus, equations (21), (22) provide a tool for analysis of the dependence of the local susceptibility $X_{\chi}\left(\zeta \mid x_{t}, x_{h}\right)$ with respect to the parameters $x_{t}$ and $x_{h}$.

Definition (8) in scaling variables reads

$$
\bar{X}_{\chi}\left(x_{t}, x_{h}\right)=\int_{0}^{1} X_{\chi}\left(\zeta \mid x_{t}, x_{h}\right) d \zeta .
$$

\section{Results}

\subsection{Plots of the order parameter profile}

Typical examples of the order parameter profiles in the case of Neumann - infinity boundary conditions are shown in Fig. 1. Here, Fig. 1(a) is for $x_{t}=-8.45, x_{h}=-15.63$, and in this case there exists only one order parameter that satisfies the boundary conditions, and it takes positive values only. Fig. 1(b) represents the case when a new order parameter that satisfies equation (14) and boundary conditions (9) appears. In this case, the blue order parameter is of lower energy meaning that the system is in a liquid state. For a bit lower values of the ordering field, three distinct profiles exist, that are illustrated in Fig. 1(c). Here, the profile of lowest energy is also the blue one, and it indicates that the system is in gas state near the left boundary and in the liquid state in the right one. Further lowering the ordering field causes the system to goes back to a state with a single order parameter, see the case Fig. 1(d), taking both - negative and positive values. Consequently, lake the case in Fig. 1(c), here the system is in gas state near the left boundary and in liquid state near the right one.
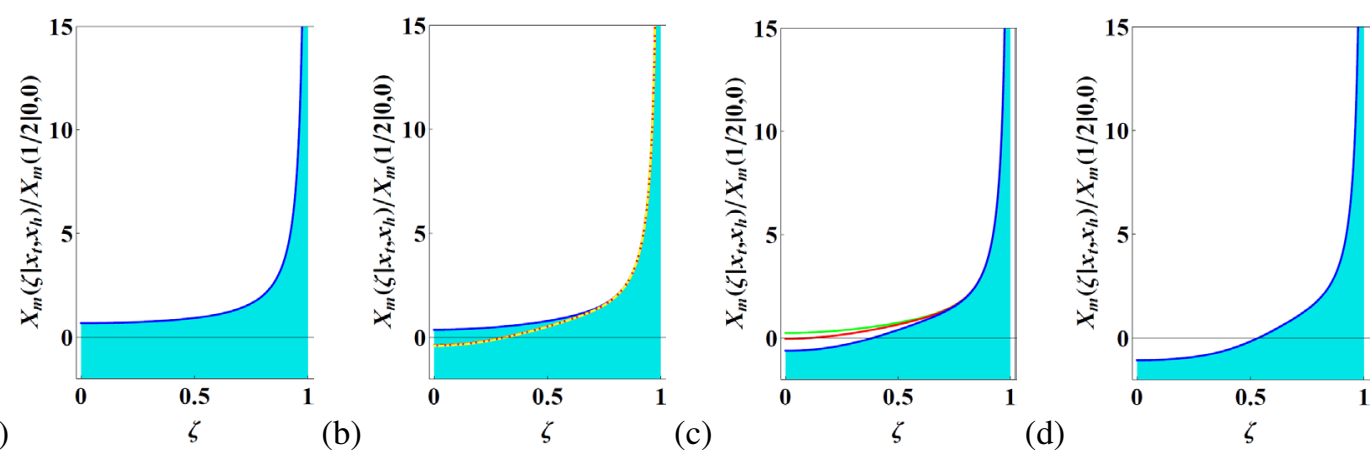

Figure 1. Order parameter profiles in the cases $x_{t}=-8.45$, and: $x_{h}=-15.63(\mathrm{a}), x_{h}=-30.12(\mathrm{~b}), x_{h}=-32(\mathrm{c})$ and $x_{h}=-62.5(\mathrm{~d})$. 


\subsection{Plot of the phase diagram}

The phase diagram is shown on Fig. 2. It is a straight line starting from $x_{t}=-6.42458$ and $x_{h}=$ -29.5044. The four dots on Fig. 2 indicate the cases in Fig. 1, namely: the most right black dot is Fig. 1(a), the green one is Fig. 1(b), the blue dot is Fig. 1(c), and the most left black dot is Fig. 1(d).

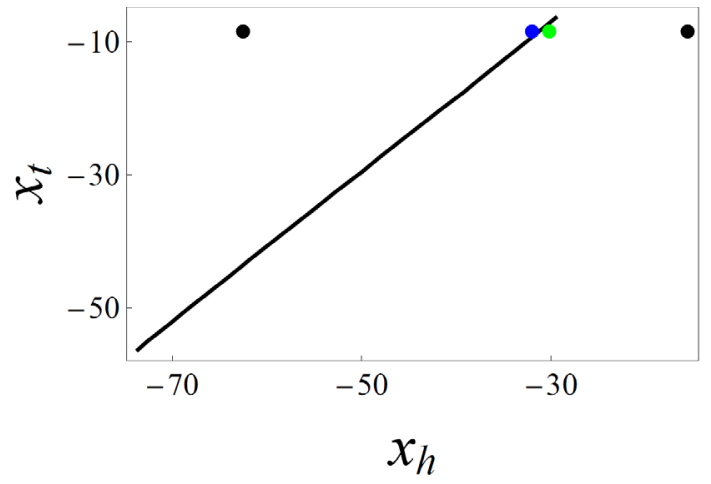

Figure 2. Phase diagram for the Neumann - infinity case. Dots indicate the four cases in Fig. 1.

Our observation is that there exists a short segment in the phase diagram between its top point $T_{c L}\left(x_{t}=-6.42458, x_{h}=-29.5044\right)$ and the point $T_{c a p}\left(x_{t}=-6.61822, x_{h}=-29.67\right)$, crossing which the system jumps between a less dense gas and a more dense gas. Thus, the very capillary condensation point is $T_{\text {cap }}$, bellow which system jumps between a gas and a liquid states. The same findings concerning the case of $(+,+)$ boundary conditions are reported in [15].

\subsection{Plots of the local susceptibility}

Fig. 3 represents several examples of profile curves of the local susceptibility. It is seen, that the profile curve of the local susceptibility corresponding to $x_{t}=-8.45, x_{h}=-62.50$ is not a monotonic one, whereas the profile curves of the local susceptibility corresponding to $x_{t}=-8.45$ and $x_{h}=-15.63$, $x_{h}=-32.00$ are monotonic.

\subsection{Plots of the total susceptibility}

Finally, two plots of the scaling function of the total susceptibility (23) are shown in Fig. 4. The left figure shows the appearance of the total susceptibility near the point $T_{c L}\left(x_{t}=-6.42458, x_{h}=\right.$ -29.5044), where several solutions of Eq. (14) exists. The right figure represents the classic point of capillary condensation $T_{\text {cap }}\left(x_{t}=-6.61822, x_{h}=-29.67\right)$. It should be noted, that when the system traverses the segment in the phase diagram between $T_{c L}$ and the capillary condensation point $T_{c a p}$, the system jumps between a states of a less dense gas and a more dense gas. Below the capillary condensation point, the system jumps between gas and liquid states.

\section{Conclusions}

The behaviour of the local and total susceptibilities of a fluid system in a film geometry are studied. The Ginsburg-Landau Ising type model and Neumann-infinity boundary conditions are employed. 

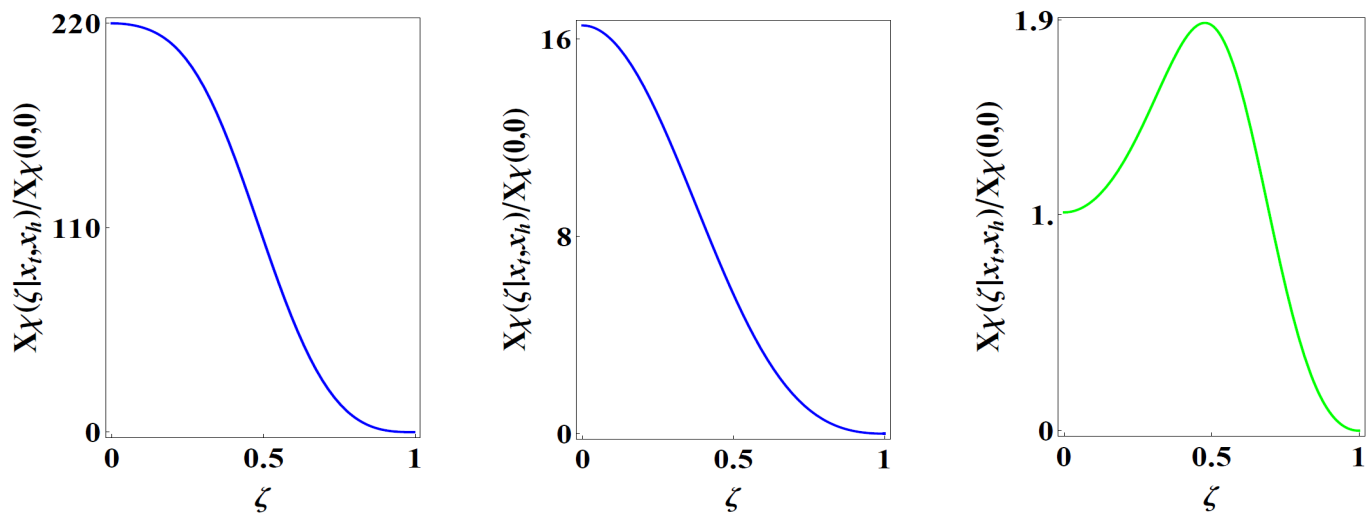

Figure 3. Local susceptibility profiles in the cases $x_{t}=-8.45$, and: $x_{h}=-30.12$ (left), $x_{h}=-32$ (centre), and $x_{h}=-62.5$ (right).
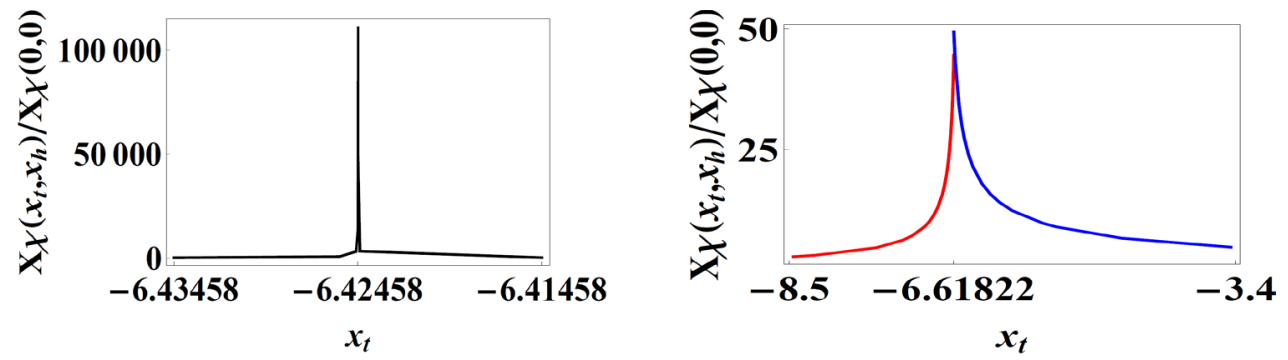

Figure 4. The temperature behavior of the total susceptibility near the point $T_{c L}$, where the system experiences jumps between states of a less dense gas and a more dense gas (left), and the classic point of a beginning of the capillary condensation $T_{c a p}$ (right).

Exact analytic results for the order parameter, as well as local and total susceptibilities are presented. Plots of the order parameter profiles, phase diagram, and local susceptibilities profiles are presented. In this problem, the fluid system is always in a liquid state at the right boundary, and achieves gas of liquid states near the left boundary, depending on the values of the parameters $x_{t}$ and $x_{h}$ (see, e.g. $[17,18])$.

It is found that the phase diagram is an approximately straight line, starting from the point $T_{c L}\left(x_{t}=\right.$ $-6.42458, x_{h}=-29.5044$ ), see Fig. 2. As it is seen in Fig. 4 (left), the total susceptibility there achieves extremely high values and theoretically diverges.

Our observation is that below the point $T_{\text {cap }}\left(x_{t}=-6.61822, x_{h}=-29.67\right)$, the system jumps between a gas and a liquid states. This means, that $T_{\text {cap }}$ is the true capillary condensation point, but the total susceptibility achieves finite values there, see Fig. 4 (right). The total susceptibility diverges at the point $T_{c L}$, and along the segment starting from $T_{c L}$ and ending to $T_{c a p}$, the system jumps between a less dense gas and a denser one. 


\section{References}

[1] K. Binder, Critical Behaviour of Surfaces; vol 8 in Phase Transitions and Critical Phenomena, eds C. Domb and J. L. Lebowitz (Academic, London, 1983)

[2] H. W. Diehl, Field-theoretical approach to critical behavior of surfaces; vol 10 in Phase Transitions and Critical Phenomena, eds C. Domb and J. L. Lebowitz (New York: Academic, 1986)

[3] R. Evans, J. Phys.: Condens. Matter 2, 8989 (1990)

[4] M. Tröndle, L. Harnau and S. Dietrich, J. Chem. Phys. 129, 124716 (2008)

[5] Z. Borjan and P. Upton, Phys. Rev. E 63, 065102 (2001)

[6] R. Evans and U. M. B. Marconi, J. Chem. Phys. 84, 2376-99 (1986)

[7] R. Okamoto and A. Onuki, J. Chem. Phys. 136, 114704 (2012)

[8] D. Dantchev, F. Schlesener and S. Dietrich, Phys. Rev. E 76, 011121 (2007)

[9] A. Drzewiński, A. Maciołek, A. Barasiński and S. Dietrich, Phys. Rev. E 79, 041145 (2009)

[10] J. Cahn, J. Chem. Phys. 66, 3667-72 (1977)

[11] P.-G. de Gennes, Rev. Mod. Phys. 57, 827-63 (1985)

[12] F. P. Toldin and S. Dietrich, J. Stat. Mech. 11, P11003 (2010)

[13] D. Dantchev, J. Rudnick and M. Barmatz, Phys. Rev. E 75, 011121 (2007)

[14] T. W. Burkhardt and H. W. Diehl, Phys. Rev. B 50 3894-3898 (1994)

[15] D. Dantchev, V. Vassilev and P. Djondjorov, Journal of Statistical Mechanics: Theory and Experiment 2015, P08025 (2015)

[16] E. T. Whittaker and G. N. Watson, A Course of Modern Analysis (Cambridge University Press, London, 1963)

[17] R. Evans, Liquids at interfaces (Elsevier, Amsterdam, 1990)

[18] H.-J. Butt, K. Graf and M. Kappl, Contact Angle Phenomena and Wetting; Chapter 7 in Physics and Chemistry of Interfaces (Wiley-VCH Verlag \& Co. KGaA, Weinheim (FRG), 2003) 